\title{
Wann kostspielige Therapien kein Problem sind
}

\author{
Immer wieder kommt es wegen Behandlungen mit teueren Arzneien zu Kon- \\ flikten zwischen Patienten, Kassen und Ärzten. Dabei muss sich der Arzt in vielen \\ Fällen gar keine Sorgen machen. Ein Fachanwalt erklärt die Hintergründe.
}

- Gerade bei seltenen Erkrankungen sind Ärzte oft unsicher, ob die Kasse die Therapiekosten übernimmt, zumal in solchen Fällen oft hohe Kosten entstehen. Ein Regress soll tunlichst vermieden werden. Dabei sind Ärzten die Hände gar nicht so stark gebunden wie oft vermutet. Das zeigt ein Beispiel anhand eines Falles von Mucopolysaccharidose VI.

Dabei handelt es sich um eine sehr seltene lysosomale Speicherkrankheit. In Deutschland leidet durchschnittlich ein Neugeborenes pro Jahr darunter. Zur Behandlung ist seit 2006 eine Enzymersatztherapie, (Galsulfase, Naglazyme ${ }^{\circ}$ ), zugelassen. Die Jahrestherapiekosten erreichen sechsstellige Beträge, bei Erwachsenen auch eine Million Euro und mehr.

\section{Der Fall:}

Der zur Zeit der Zulassung dieser Therapie bereits deutlich über 30-jährige Patient leidet an einer relativ milden Verlaufsform von Mucopolysaccharidose. Er zeigt typische Symptome wie multiple Kontrakturen, mehrfache Hüftgelenksendoprothesen, Herzklappenveränderungen und Hornhauttrübung.

Für seinen Arzt war die Indikation zur Galsulfase-Therapie offensichtlich. Die Klinikleitung verlangte eine Absicherung. Der im Februar 2007 gestellte Kostenübernahmeantrag wurde abgelehnt. Das Widerspruchsverfahren zog sich über zwei Jahre. Die Kasse argumentierte, in die Zulassungsstudien seinen nur Patienten zwischen 5 und 29 Jahren einbezogen gewesen, womit keine hinreichende Evidenz für die Wirksamkeit bei einem über 30-jährigen Patienten vorliege.

\section{Langwierige Entscheidungsprozesse}

Die Entscheidung im Sommer 2011 ergab keinen Zweifel an der Leistungspflicht der Kasse. Der Richter wollte die Therapie aber zur Evaluation zunächst auf drei Monate begrenzen (SG Münster, Az.: S 16 (11) KR 78/09). Da dies nicht sinnvoll war, weil eine Enzymersatztherapie nicht binnen dreier Monate belastbare Ergebnisse zur individuellen Wirksamkeit ergeben kann, wurde eine Berufung zum Landessozialgericht Nordrhein-Westfalen erforderlich (Az.: L 5 KR 601/11).

In der Verhandlung (September 2012) wurden die LSG-Richter deutlich: Es sei völlig unverständlich, dass ein Patient, der ganz offensichtlich dem zugelassenen Anwendungsgebiet der einzig verfügbaren Therapie unterfalle, mehr als fünf Jahre unbehandelt bleibe, weil sich die Ärzte nicht trauten, die offensichtlich rechtmäßige Verordnung auf Kassenrezept vorzunehmen und weil die Kasse mit offensichtlich unbegründeten Argu-

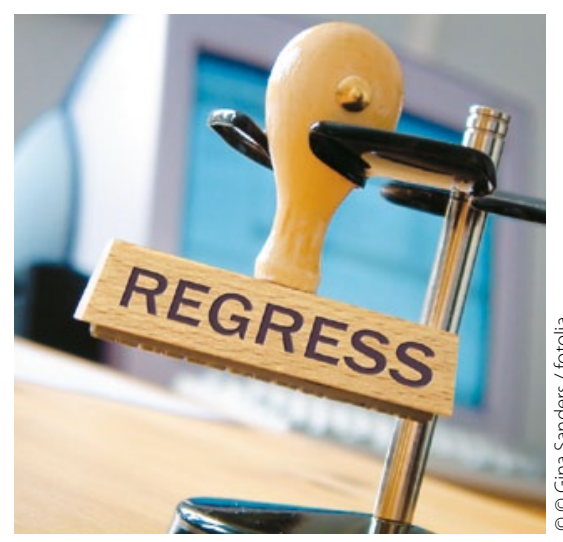

Der Regress: für viele Ärzte eine allgegenwärtige, kaum durchschaubare Bedrohung. menten den Behandlungsbeginn hinauszögere.

\section{Schadensersatzanspruch bei Therapieverzögerung?}

Die Richter deuteten an, der Patient habe möglicherweise einen Schadensersatzanspruch zum Ausgleich in der Zwischenzeit entstandener weiterer Gesundheitsschäden. Die Kasse sagte die Kostenübernahme zu.

Gemäß \$\$2, 12, 27, 31, 34 SGB V haben GKV-Patienten Anspruch auf Arzneimittel, die nicht aus der Versorgung ausgeschlossen sind, sofern die Behandlung dem allgemein anerkannten Stand der medizinischen Erkenntnis und dem Wirtschaftlichkeitsgebot entspricht. Ist eine Arzneitherapie alternativlos, entspricht sie mit der Zulassung dem medizinischen Stand.

Auch das Wirtschaftlichkeitsgebot steht der Verordnung teuerer Therapien nur entgegen, wenn das angestrebte Therapieziel kostengünstiger erreicht werden kann. Besteht ein medizinischer Anlass zur Verordnung einer teueren Therapie, ist diese auch wirtschaftlich (BSG, Az.: B 6 KA 41/03). Die Abwägung des Kosten-Nutzen-Verhältnisses einer Therapie gehört nicht zu den Aufgaben des verordnenden Arztes (BSG, Az.: B 6 KA 13/05 R).

Da der Patient dem zugelassenen Anwendungsgebiet von Naglazyme ${ }^{\circ}$ unterfiel, hatte er einen Anspruch darauf. Das Argument, die Zulassungsstudien ergäben keine Evidenz für Patienten jenseits der 30 mit milderen Verlaufsformen, überzeugt nicht.

Fazit: Gibt es nur eine Therapieoption, ist diese auch wirtschaftlich, egal was sie kostet! Im Übrigen geben die Sozialgerichte Raum für eine Klärung der Leistungspflicht nur im Vorfeld des potenziell umstrittenen Off-label Use.

GERHARD NITZ : 